\section{Tabagismo e câncer no Brasil: evidências e perspectivas}

\section{Tobacco smoking and cancer in Brazil: evidence and prospects}

Victor Wünsch Filho'

Antonio Pedro Mirra'

Rossana V. Mendoza López'

Leopoldo F. Antunes"

' Faculdade de Saúde Pública da Universidade de São Paulo (USP)

"Escola de Artes, Ciências e Humanidades da Universidade de São Paulo (USPLeste)

Correspondência: Victor Wünsch Filho. Departamento de Epidemiologia da Faculdade de Saúde

Pública da Universidade de São Paulo, Av. Dr. Arnaldo, 715. E-mail: wunsch@usp.br

\section{Resumo}

Neste artigo analisa-se a tendência temporal da prevalência do tabagismo no Brasil, bem como as assimetrias da prevalência de acordo com as regiões do país, a idade, o gênero e o nível socioeconômico da população. Desde o estabelecimento da relação entre tabagismo e câncer de pulmão há 60 anos, o número de tumores malignos com evidências de associação causal com o tabagismo ascendeu a vinte. O declínio da prevalência do tabagismo na população brasileira tem sido constante desde o final da década de 80 . Até 2003, foi mais intenso entre os homens. A partir daquele ano, a queda tornou-se mais pronunciada entre as mulheres. As mais altas prevalências de tabagismo encontram-se no Sudeste e Sul, as duas regiões com maiores incidências de neoplasias estritamente relacionadas ao tabaco (cavidade oral, esôfago e pulmão). A exposição ambiental à fumaça do tabaco também foi examinada considerando-se os efeitos para os adultos não fumantes, que apresentam maior risco de tumores de pulmão, laringe e faringe, e entre crianças de pais fumantes, suscetíveis ao risco de hepatoblastoma e leucemia linfocítica aguda. Apesar do incontestável sucesso da política de controle do tabagismo no país, as ações de prevenção devem considerar que as parcelas da população com piores condições socioeconômicas e com baixo nível educacional são as que apresentam taxas mais altas de prevalência de tabagismo. Dentro destes segmentos populacionais os adolescentes representam uma prioridade.

Palavras-chave: Brasil. Prevalência de tabagismo. Incidência de câncer. Tumores tabaco-relacionados. Tendência temporal do tabagismo. 


\section{Abstract}

In this paper, the prevalence of smoking in Brazil is examined according to time trend and regions of the country, as well as considering the asymmetries of the prevalence of tobacco smoking by age, sex and socioeconomic level of the population. Since the establishment of the relationship between smoking and lung cancer 60 years ago, the number of malignant tumors with evidence of a causal association with tobacco smoking has amounted to twenty. From the late 80's, the decline of the prevalence of smoking in the Brazilian population has been continuous. Until 2003, the decrease was more significant among men, when an inversion occurred and the decline became more pronounced in women. The highest prevalence of smoking is detected in the Southeast and South, and these two regions present the highest incidence of tumors closely related to tobacco (oral cavity, esophagus and lung). Environmental exposure to tobacco smoke was also examined considering the effect in nonsmoking adults, with a higher risk of lung, larynx and pharynx tumors, as well as among children of smoking parents more susceptible to the risk of hepatoblastoma and acute lymphocytic leukemia. Despite the undeniable success of the policy to control tobacco smoking in the country, health professionals should be attentive to populations with low socioeconomic status and lower educational levels who show the highest prevalence rates of smoking. Adolescents represent a priority in these populations.

Keywords: Brazil. Prevalence of smoking. Incidence of cancer. Tobacco-related tumors. Trends in tobacco smoking.

\section{Introdução}

Atualmente mais de um bilhão de pessoas são fumantes no mundo e na década de 2030 estima-se que esse total poderá chegar a dois bilhões. A maioria destes fumantes estará nos países em desenvolvimento ${ }^{1}$. O impacto sobre a saúde decorrente do uso do tabaco é bem conhecido: responsável por $90 \%$ dos tumores pulmonares, $75 \%$ das bronquites crônicas, $25 \%$ das doenças isquêmicas do coração²

Desde a primeira relação estabelecida entre consumo de cigarros e câncer de pulmão no início da década de 50 do século XX, os estudos epidemiológicos têm continuamente identificado novas localizações de câncer com vinculações causais com o uso do tabaco, que hoje ascende a 20 diferentes tipos de tumor ${ }^{3}$. Mesmo os não fumantes expostos à fumaça do tabaco nos ambientes que vivenciam no dia a dia estão sob maior risco de desenvolverem câncer.

Medidas restritivas de comercialização e consumo do tabaco nos países desenvolvidos levaram a indústria do tabaco a direcionar seus esforços de venda para países mais pobres, onde havia perspectivas de crescimento do consumo e contínua reposição de fumantes. Os alvos principais de suas campanhas promocionais têm sido os adolescentes e jovens. Assim, no final da década de 90, dos cerca de 100 mil jovens que se iniciavam no tabagismo a cada dia no mundo, $80 \%$ residiam em países em desenvolvimento ${ }^{4}$.

Para examinar as mudanças de comportamento da população brasileira em relação ao tabagismo e as implicações destas mudanças na ocorrência de câncer, esta análise está dividida em três partes. Em primeiro lugar, a especificidade da dinâmica do consumo de tabaco no Brasil, considerando-se a tendência da prevalência no tempo e por regiões do país, bem como sua distribuição por idade, gênero, e estratos socioeconômicos. Em seguida, será analisada a prevalência da exposição à fumaça ambiental derivada do tabaco. Por último, 
serão examinadas as evidências atuais da associação do uso do tabaco e câncer, e as perspectivas da incidência e da mortalidade pela doença no país.

\section{Dimensão do tabagismo no Brasil}

Em 2008, o Instituto Brasileiro de Geografia e Estatística (IBGE), em conjunto com o Instituto Nacional do Câncer (INCA), conduziu o mais recente inquérito sobre tabagismo em maiores de 15 anos de idade 5 . Foi constado que $17,2 \%$ da população brasileira fazia uso regular de tabaco, equivalente a 25 milhões de pessoas. A maior prevalência de fumantes foi detectada na região Sul (19\%), e os menores percentuais nas regiões Centro Oeste e Sudeste (16,9\%).

A adoção e o monitoramento de ações efetivas para o controle do tabagismo dependem do conhecimento da extensão do problema em diferentes subgrupos da população, bem como da avaliação de sua tendência no tempo. No Brasil, os inquéritos populacionais para avaliar a prevalência do consumo de tabaco existem há duas décadas. Além do mais recente, em 2008, foi realizada em 1989 a Pesquisa Nacional de Saúde e Nutrição, primeiro inquérito de base populacional no país que incluiu informações sobre consumo de tabaco entre indivíduos com 15 ou mais anos de idade $^{6}$. Em 2003, o Instituto Nacional do Câncer (INCA) conduziu outro inquérito de base populacional abrangendo indiví- duos de ambos os sexos com 15 ou mais anos de idade, com o objetivo de conhecer a prevalência de diferentes fatores de risco ambientais para câncer ${ }^{7}$. Além desses grandes inquéritos nacionais, há alguns estudos em populações delimitadas a determinadas regiões, a exemplo dos inquéritos realizados no município de São Paulo no início das décadas de $1990^{8}$ e $2000^{9}$.

\section{Tendência no tempo}

Os dados da Tabela 1 mostram que no período entre 1989 e 2008 ocorreu acentuado recuo da prevalência de fumantes no país em ambos os sexos. Na cidade de São Paulo, o declínio de fumantes entre os homens e mulheres também foi expressivo entre 1990 e 2002. A tendência de queda do tabagismo mais acentuada entre mulheres em relação aos homens contrasta com o observado na cidade do Rio de Janeiro entre os anos de 1989 e $2001^{10}$, onde a queda foi menos acentuada entre as mulheres do que entre os homens.

O quadro da prevalência do tabagismo na população brasileira entre 1989 e 2003 foi bem caracterizado por Monteiro et al. ${ }^{11}$. Os autores observaram uma queda total da ordem de $35 \%$, ou seja, uma redução média de 2,5\% ao ano, mais acentuada na população masculina. Porém, os dados do inquérito de 2008 indicam que houve inversão desta tendência a partir de 2003, período em que o declínio da prevalência

Tabela 1 - Prevalência de consumo de tabaco no Brasil e no Estado de São Paulo, em homens e mulheres, em diferentes períodos.

Table 1 - Prevalence of tobacco smoking in Brazil and in the city of São Paulo, men and women, in different time periods.

\begin{tabular}{|c|c|c|c|c|}
\hline \multirow[t]{2}{*}{ População/Ano } & \multirow[t]{2}{*}{ Faixa etária } & \multirow[t]{2}{*}{ Referência } & \multicolumn{2}{|c|}{ Prevalência } \\
\hline & & & Homens & Mulheres \\
\hline \multicolumn{5}{|c|}{ Inquéritos populacionais no Brasil } \\
\hline 1989 & 15 anos ou mais & Brasil, $1990^{6}$ & 40,3 & 26,2 \\
\hline 2003 & 15 anos ou mais & INCA, $2004^{7}$ & 27,0 & 18,4 \\
\hline 2008 & 15 anos ou mais & IBGE, $2008^{5}$ & 21,6 & 13,1 \\
\hline \multicolumn{5}{|c|}{ Inquéritos populacionais na cidade de São Paulo } \\
\hline 1990 & $15-59$ anos & Rego et al., $1990^{8}$ & 44,6 & 31,9 \\
\hline $2001-2$ & 15-59 anos & Marcopito et al., $2005^{9}$ & 25,4 & 19,9 \\
\hline
\end{tabular}


do tabagismo tornou-se mais intenso entre as mulheres $(28,8 \%)$ do que entre os homens (20\%) (Tabela 1).

Entre 1989 e 2003, o declínio da prevalência total de tabagismo foi distinto entre as capitais do país, mais expressivo nas cidades de Manaus (45,2\%) e Rio de Janeiro $(43,3 \%)$. Porto Alegre apresentou a menor queda no período $(10,3 \%)^{7}$.

\section{Idade, gênero e tabagismo}

A idade é uma variável fundamental na epidemiologia do câncer. O período de latência, período de tempo compreendido entre a data da primeira exposição e a data do diagnóstico, é bastante longo em câncer, cerca de três décadas no caso do início da exposição à fumaça de tabaco e a ocorrência do câncer de pulmão ${ }^{12}$.

O tabagismo, bem como o uso de outras drogas aditivas, tende a se estabelecer durante a adolescência. Quanto mais precoce a idade do início, maior a probabilidade do indivíduo tornar-se dependente da nicotina $^{13}$. Como conseqüência, os adolescentes fumantes sofrem um expressivo risco de virem a desenvolver algum tipo de câncer tabaco-relacionado ainda na vida adulta produtiva, com grande repercussão em termos de anos de vida perdidos para a sociedade.

Fatores ambientais, sociodemográficos, comportamentais e pessoais estão relacionados ao uso de tabaco pelos adolescentes. O tabagismo na adolescência tem sido associado ao hábito de fumar dos pais, amigos e irmãos com maior idade. Baixo rendimento nos estudos, abandono da escola e trabalho remunerado, são fatores também conectados ao tabagismo na adolescência. Desajuste familiar, separação dos pais e moradia com outras pessoas que não os pais biológicos, a baixa auto-estima e a depressão são condições que aumentam a probabilidade da fixação do tabagismo entre os adolescentes. Deve-se assinalar ainda como facilitador para a iniciação a limitada percepção entre os adolescentes do risco decorrente do tabagismo ${ }^{14-18}$.
Desde a introdução do tabaco na sociedade moderna, após o descobrimento da América, e a expansão de seu consumo durante a Revolução Industrial, o tabagismo tem sido uma experiência predominantemente masculina. Porém, com os movimentos de liberação feminina e da maior integração das mulheres à lógica de produção capitalista durante as décadas de $60 \mathrm{e}$ 70 do século XX, observou-se o incremento do tabagismo entre as mulheres.

No Brasil, desde 1989, a redução do tabagismo ocorre em todas as faixas etárias, sendo principalmente evidente nas faixas etárias mais jovens, abaixo dos 45 anos nos homens e dos 35 anos nas mulheres ${ }^{5,11}$.

Estudo em Londrina, Paraná, realizado em 2003 com escolares do Ensino Médio entre 15 a 18 anos de idade, identificou que $15,4 \%$ dos estudantes do sexo feminino e $20,9 \%$ do sexo masculino eram tabagistas. Em média, a quantidade de cigarros consumidos por dia foi mais elevada entre os rapazes (9,2 cigarros/dia) do que entre as moças (5,6 cigarros/dia). No conjunto dos estudantes fumantes, $15 \%$ relataram ter experimentado cigarro pela primeira vez antes dos 14 anos de idade. Apenas 3\% dos adolescentes fumantes admitiram terem se iniciado no tabagismo após os 16 anos de idade ${ }^{19}$.

Os dados do Vigescola ${ }^{20}$, estudo com escolares de 12 a 16 anos de idade, revelam que nas capitais dos estados do Norte e Nordeste a prevalência de tabagismo é maior entre os meninos, porém nas capitais dos estados do Sudeste e Sul a prevalência é mais elevada entre as meninas.

\section{Condições socioeconômicas e tabagismo}

No início da expansão do tabagismo, as pessoas de classes sociais abastadas apresentavam níveis mais altos de consumo. Gradativamente esta situação se modificou e hoje as parcelas da população com mais baixa renda estão mais expostas ao tabaco. Na Noruega, a prevalência de tabagismo entre homens de mais alta renda diminuiu de $75 \%$ em 1955 para $28 \%$ em 1990, mas 
entre os de baixa renda a redução foi menos expressiva, $60 \%$ para $48 \%{ }^{4}$.

Na Nova Zelândia, Whitlock et al. ${ }^{21}$ identificaram um gradiente socioeconômico no consumo de tabaco. A prevalência de tabagismo foi $12 \%$ entre aqueles com educação universitária e 35\% entre pessoas com menos de dois anos de estudo. A estratificação da população por faixas de renda e ocupação revelou consumo mais elevado, de duas a três vezes, nos grupos com piores condições sociais e econômicas. Além disso, baixa renda, ocupação em atividades com menor qualificação e restrita escolaridade foram fatores preditivos de falhas nas tentativas de abandono do tabagismo e de maior quantidade de cigarros fumados. A correlação entre níveis mais elevados de tabagismo com baixa renda, pior nível educacional e ocupações precárias repete-se nas pesquisas realizadas em diferentes países ${ }^{22-28}$.

Em 1987, Lolio et al. ${ }^{29}$ investigaram em Araraquara, no Estado de São Paulo, as diferenças na prevalência de fumantes entre estratos socioeconômicos. Entre homens analfabetos ou com menos de quatro anos de estudo formal, a prevalência de tabagismo atingiu $48,6 \%$, enquanto nos indivíduos com curso superior completo a prevalência foi $35,7 \%$. Tendência análoga, mas com porcentagens inferiores, foi observada entre as mulheres. Nos anos 1990, em Porto Alegre, foi verificada maior prevalência de tabagismo entre homens que recebiam menos de dois salários mínimos, com menos de cinco anos de estudo e exercendo ocupações menos qualificadas ${ }^{30}$. Dados equivalentes foram notados em Belo Horizonte mais recentemente ${ }^{31}$. Na cidade de São Paulo também foi observada relação inversa entre prevalência de tabagismo e nível educacional ${ }^{32}$.

Szwarcwald et al..$^{33}$ analisaram amostra representativa da população brasileira e estimaram em $30 \%$ os homens fumantes entre aqueles que dispunham menos de quatro bens no domicílio (televisão, geladeira, micro-ondas, máquina de lavar roupa, máquina de lavar louça, microcomputador, aparelho de som, telefone fixo, telefone ce- lular ou carro). Entre os que possuíam mais de oito desses bens, $21 \%$ eram fumantes.

Os resultados dos inquéritos populacionais brasileiros de 1989 e 2003 mostraram que em ambos os sexos a intensidade do declínio do tabagismo foi função direta da renda familiar ${ }^{11}$. Inquérito populacional por telefone realizado em 2006 mostrou prevalência de tabagismo mais elevada nos indivíduos com menor nível educacional ${ }^{34}$.

A influência do status socioeconômico sobre o tabagismo entre adolescentes é controversa. Há relatos de que a disponibilidade de recursos financeiros tende a influenciar o uso de tabaco pelos adolescentes ${ }^{35}$.

\section{Exposição ambiental à fumaça do tabaco}

Os danos ao organismo humano provenientes do tabagismo não afetam apenas as pessoas que fumam, mas atingem as não fumantes que vivem sob poluição pela fumaça de cigarros nos domicílios, nos ambientes de trabalho, de lazer, escolas e demais espaços públicos fechados. A fumaça inalada pelos fumantes passivos ou involuntários é responsável por grande parte das doenças tabaco-relacionadas incidentes nestes indivíduos, em particular o câncer de pulmão ${ }^{36,37}$. Esta poluição ambiental é decorrente da fumaça exalada pelo fumante (corrente primária ou principal), que corresponde a $25 \%$ do total, e por aquela resultante da queima da ponta do cigarro ou outro produto do tabaco (corrente secundária). A corrente secundária produzida pela combustão do cigarro entre as tragadas corresponde aos restantes $75 \%$ de fumaça de tabaco presente nos ambientes, sendo a mais importante fonte da poluição ambiental e com maiores concentrações de todos os componentes carcinogênicos do tabaco ${ }^{38}$.

As implicações de exposições a determinados fatores de risco ambientais em fases precoces da vida (intra-uterina e infância) para a saúde na vida adulta, inclusive ocorrência de câncer, têm sido objeto crescente de pesquisa na epidemiologia moderna ${ }^{39}$. $\mathrm{O}$ tabagismo passivo pode se iniciar na fase 
de vida intra-uterina, pois a mulher grávida que fuma, ou mesmo não fazendo uso do tabaco, mas convivendo com pessoas fumantes, pode induzir repercussões deletérias ao feto, pela condução de substâncias tóxicas através do cordão umbilical.

As residências são os locais predominantes de exposição ao tabaco ambiental. Muitas crianças são expostas precoce e diariamente à fumaça do tabaco enquanto se alimentam, brincam ou dormem. Além da residência, as crianças podem ser expostas à fumaça do tabaco na escola ou nos veículos ${ }^{40}$. O mais abrangente estudo sobre tabagismo involuntário em crianças foi realizado nos Estados Unidos ${ }^{41}$ e mostrou que a exposição diária variou de forma considerável de acordo com o status socioeconômico da família: $41 \%$ nas crianças de baixo nível socioeconômico e $21 \%$ naquelas de mais alto nível socioeconômico.

Alguns estudos indicaram que a exposição involuntária ao tabaco entre adultos está relacionada à ocupação e ao status socioeconômico. Exposições intensas são comuns entre adultos empregados em trabalhos nas linhas de produção industrial, e em ocupações envolvendo trabalhadores com salários e níveis educacionais mais baixos $^{42-44}$.

Elevadas taxas de material particulado em suspensão pela fumaça do cigarro afetam até $80 \%$ dos trabalhadores nos seus ambientes de trabalho, principalmente quando atuam em restaurantes, hotéis, bares, boates e bingos $^{38}$. A presença de substâncias cancerígenas na urina de trabalhadores da saúde não fumantes expostos à poluição pela fumaça do cigarro nos ambientes laborais explicaria o aumento do risco de câncer de pulmão encontrado nesses trabalhadores ${ }^{45}$.

No inquérito realizado na população brasileira em 2008, a fumaça do cigarro foi referida como presente nos domicílios $(27,9 \%)$, nos ambientes de trabalho $(24,4 \%)$ e em restaurantes $(9,9 \%)^{5}$.

Estudo realizado nos anos de 2002 e 2003 em sete países da América Latina (Argentina, Brasil, Chile, Costa Rica, Paraguai, Peru e Uruguai) avaliou a concentração de nicotina no ar em diferentes espaços públicos: hospitais, escolas secundárias, edifícios de prefeituras, aeroportos, restaurantes e bares ${ }^{46}$. Em geral, os níveis de nicotina ambiental detectados nesses países foram menores do que os observados em países da Europa e da América do Norte, porém alguns resultados são preocupantes. Poluição tabágica foi detectada nos ambientes hospitalares, apesar de haver nos países pesquisados legislação nacional ou local restringindo ou banindo o uso do tabagismo nestes locais; esta é, portanto, uma indicação de que a legislação não é seguida e que os profissionais da saúde não têm completa adesão à proposta de ambientes livres de tabaco nos hospitais. Considerando-se os possíveis efeitos da fumaça do tabaco para adequada recuperação dos pacientes, a exigência de ambientes livres de tabaco deveria fazer parte dos critérios para o processo de credenciamento dessas instituições.

Em muitas das escolas secundárias dos sete países latino-americanos pesquisados foram detectados níveis elevados de nicotina ambiental, embora o tabagismo tenha sido banido das escolas na maioria desses países.

Hospitais e escolas são espaços críticos para a política de ambientes livres da fumaça do tabaco, pois os profissionais de saúde, assim como os professores e trabalhadores em educação, podem irradiar modelos de comportamento para a população como um todo e, particularmente, para os adolescentes e jovens.

Entre os países estudados, o Brasil apresentou a maior média de exposição à nicotina ambiental em restaurantes ${ }^{46}$. $O$ estudo revelou que, mesmo em áreas reservadas a não fumantes, as concentrações de nicotina ambiental são altas. Estes resultados corroboram as evidências de outros estudos de que estabelecer áreas de não fumantes é uma medida inócua para controlar a exposição à fumaça ambiental do tabaco ${ }^{47,48}$. A contínua exposição em restaurantes e bares a concentrações de nicotina ambiental põe em risco a saúde de trabalhadores nesses ambientes e indica a necessidade de medi- 
das para livrar do tabaco todos os espaços de trabalho.

A prevenção das doenças tabacorelacionadas entre pessoas não fumantes deve ser o foco de programas antitabaco, pois esta ação repercute tanto no tabagismo passivo quanto no tabagismo ativo. As iniciativas para a obtenção de ambientes livres da fumaça do tabaco devem visar o não fumar em qualquer ambiente fechado.

\section{Tabagismo e câncer}

O tabagismo é, isoladamente, a principal causa de câncer no mundo. Desde o clássico estudo de Doll e Hill ${ }^{49}$, que identificou a estreita associação entre consumo de cigarros e câncer de pulmão, novas evidências foram acumuladas para outros tumores malignos (Tabela 2). De acordo com a última revisão conduzida pela Agência Internacional de Pesquisas em Câncer (International Agency for Research on Cancer - IARC), Parte E do Volume 100 das Monografias da IARC (não publicada), sobem a 20 os tumores malignos associados com o tabagismo ${ }^{3}$, incluindo o câncer de ovário ${ }^{53}$ e o de cólon ${ }^{54}$. Nesta última revisão da IARC foram consideradas evidências apenas limitadas da relação do tabagismo com o câncer de mama ${ }^{3}$.

A diminuição da prevalência do tabagismo observada no país, mais acentuada entre os homens até 2003, levou à queda da mortalidade por câncer de pulmão. Entre os homens brasileiros de 30 a 49 anos, a mortalidade por câncer de pulmão é decrescente desde 1980 (primeiro ano retroativo com dados de mortalidade disponíveis); nos homens de 50 a 59 anos, o decréscimo é perceptível desde o final da década de 80; na faixa etária de 60 a 69 anos, o declínio ocorre desde 1995. Contudo, nos homens com 70 ou mais anos, a curva de mortalidade por câncer de pulmão continua ascendente ${ }^{55}$. Como esperado, a influência do declínio do tabagismo sobre a mortalidade por câncer de pulmão é observada inicialmente nas coortes de homens mais jovens. A curva ascendente da mortalidade por câncer de pulmão nas coortes masculinas mais idosas configura o resíduo da experiência passada de tabagismo. Entre as mulheres, a tendência da mortalidade por câncer de pulmão no país apresentou curvas ascendentes em todas as faixas etárias ${ }^{55}$. Esta situação reflete a ampliação do número de mulheres fumantes desde os anos 1960-1970.

No Brasil, os inquéritos populacionais indicaram que as regiões Sudeste e Sul comportam as maiores prevalências de tabagismo, em particular no meio urbano ${ }^{5,7}$. Estas duas regiões também registraram as mais altas incidências de neoplasias mais estritamente relacionadas ao tabaco.

Tabela 2 - Evolução das localizações de câncer com relação causal com o tabagismo.

Table 2 - Development of different cancer sites causally associated with tobacco smoking.

\begin{tabular}{l} 
Publicação (Ano) \\
\hline Doll \& Hill $(1957)^{50}$ \\
Doll \& Peto (1981) \\
IARC Monographs, Volume 38 (IARC 1986)
\end{tabular}

IARC Monographs, Volume $83\left(\right.$ IARC 2004) ${ }^{40}$

IARC Monographs, Volume 100, parte E, 2010 (Secretan et al. 2009) ${ }^{3}$

\section{Tumores para os quais há evidências suficientes}

\section{Pulmão}

Pulmão, boca, faringe, laringe e esôfago, pâncreas, rins, bexiga

Trato respiratório, trato digestivo superior, pâncreas, pelvis renal, bexiga

Pulmão, boca, naso-, oro-, e hipofaringe, cavidade nasal e seios paranasais, laringe, esôfago (adeno e espinocelular), estômago, pâncreas, fígado, rins (corpo e pélvis), uretra, bexiga, colo uterino, medula óssea

Pulmão, boca, naso-, oro-, e hipofaringe, cavidade nasal e seios paranasais, laringe, esôfago (adeno e espinocelular), estômago, colorretal, pâncreas, fígado, rins (corpo e pélvis), uretra, bexiga, colo uterino, ovário_(mucinoso), medula óssea (leucemia mielóide) 
O Instituto Nacional do Câncer (INCA) realiza estimativas de incidência de câncer no país comparando os dados de mortalidade e de incidência observados nos quatro registros incluídos na tradicional publicação da IARC, Cancer Incidence in Five Continents (Volume IX: Brasília, Cuiabá, Goiânia e São Paulo) $)^{56}$, e de outros 15 registros de câncer de base populacional, e projetando as mesmas proporções para as demais regiões de cada Estado ${ }^{57}$. Nas Tabelas 3 e 4 observam-se as estimativas de incidência do câncer de cavidade bucal, de esôfago e de pulmão, principais tumores malignos relacionados ao consumo de tabaco em homens e mulheres em 2008. As estimativas divulgadas pelo INCA são taxas brutas de incidência, portanto, não foi considerada a estrutura etária das populações das diferentes regiões. Por outro lado, estima-se haver discrepância da qualidade dos dados de mortalidade entre as capitais e as cidades mais afastadas nas regiões mais pobres do país, o que pode reduzir artificialmente a incidência por tipos específicos de câncer. Estes fatores isoladamente ou em conjunto podem ter importante influência nas estimativas.

Com o objetivo de balizar as estimativas brutas do INCA, levantaram-se as taxas de incidência padronizadas publicadas no Cancer Incidence in Five Continents, Volume IX ${ }^{56}$, dos tumores de cavidade bucal, de esôfago e pulmão em duas cidades: Goiânia para comparação com as estimativas para o Centro Oeste; e São Paulo para comparação com o Sudeste (Tabela 5). Nota-se que as taxas padronizadas da incidência destes três tumores tabaco-relacionados nestas duas cidades são bastante próximas das estimativas brutas do INCA para as regiões Centro Oeste e Sudeste (Tabelas 3 e 4). Assim, considerando-se as limitações assinaladas para as taxas brutas, conclui-se que é possível assumir as estimativas do INCA como boas aproximações da real incidência de câncer nas regiões brasileiras.

Os dados das Tabelas 3 e 4 permitem especular sobre certos contrastes na incidência de câncer no país. As taxas do conjunto de tumores (exceto os tumores de pele) são

Tabela 3 - Taxas brutas de incidência de câncer por 100.000 habitantes por regiões do país, com base nos dados das capitais dos estados de cada região. Homens, 2008.

Table 3 - Crude rates of cancer incidence (oral cavity, esophagus and lung) by 100,000 population in the country's geographical regions, according to data from state capitals. Men, 2008.

\begin{tabular}{lccccc}
\hline Tipo de câncer & Norte & Nordeste & Centro-Oeste & Sudeste & Sul \\
\hline Cavidade Oral & 6,3 & 10,2 & 11,8 & 18,5 & 16,9 \\
Esôfago & 3,3 & 4,8 & 7,8 & 9,6 & 13,2 \\
Pulmão & 15,4 & 16,7 & 19,0 & 30,1 & 37,2 \\
Todos (exceto pele não melanoma) & 139,8 & 173,7 & 200,7 & 297,5 & 354,5 \\
\hline
\end{tabular}

Fonte: Brasil $2007^{57}$ / Source: Brasil 2007

Tabela 4 - Taxas brutas de incidência de câncer por 100.000 habitantes por regiões do país, com base nos dados das capitais dos estados de cada região. Mulheres, 2008

Table 4 - Crude rates of cancer incidence (oral cavity, esophagus and lung) by 100,000 population in the country's geographical regions, according to the data from state capitals. Women 2008.

\begin{tabular}{lccccc}
\hline Tipo de câncer & Norte & Nordeste & Centro-Oeste & Sudeste & Sul \\
\hline Cavidade Oral & 2,7 & 4,4 & 4,6 & 5,3 & 6,2 \\
Esôfago & 0,9 & 1,8 & 2,1 & 2,6 & 3,9 \\
Pulmão & 9,9 & 9,8 & 10,5 & 15,4 & 20,9 \\
Todos (exceto pele não melanoma) & 165,0 & 201,8 & 171,9 & 316,5 & 346,7 \\
\hline
\end{tabular}

Fonte: Brasil $2007^{57}$ / Source: Brasil 2007 
Tabela 5 - Taxas padronizadas de incidência de câncer por 100.000 nas cidades de Goiânia (1999-2002) e São Paulo (1998-2002). Homens e mulheres.

Table 5 - Standardized rates of cancer incidence (oral cavity, esophagus and lung) by 100,000 in the cities of Goiânia (1999-2002) and São Paulo (1998-2002). Men and women.

\begin{tabular}{lcccc}
\hline Tipo de câncer & \multicolumn{2}{c}{ Goiânia } & \multicolumn{2}{c}{ São Paulo } \\
\cline { 2 - 5 } & Homens & Mulheres & Homens & Mulheres \\
\hline Cavidade Oral & 12,2 & 2,0 & 18,0 & 4,0 \\
Esôfago & 8,1 & 2,6 & 12,0 & 2,2 \\
Pulmão & 21,0 & 10,0 & 33.5 & 11,7 \\
\hline
\end{tabular}

Fonte: Curado et al. $2007^{56}$ / Source: Curado et al. 2007

cerca de duas vezes mais elevadas nas regiões Sudeste e Sul do que nas regiões Norte e Nordeste. Entre as diferentes regiões do país, as mais altas taxas de prevalência de tabagismo são registradas no Sudeste e Sul, regiões também com as mais elevadas incidências dos três tumores com intensa associação com o tabaco: cavidade oral, esôfago e pulmão.

Malta et al. ${ }^{55}$ mostraram que, à exceção do Estado de Minas Gerais, as regiões Sudeste e Sul apresentaram as taxas mais altas de mortalidade por câncer de pulmão. Isoladamente, o Estado do Rio Grande do Sul possui as maiores taxas de mortalidade por câncer pulmonar.

Não há explicações simples e diretas para as diferenças regionais da incidência de câncer, mas alguns fatos induzem algumas inferências. Os mais altos níveis de prevalência de tabagismo, em ambos os sexos, bem como a ocorrência das mais altas taxas de incidência e de mortalidade por câncer de pulmão no país, são constatadas em Porto Alegre ${ }^{55,57}$. O Brasil é o segundo maior produtor mundial de tabaco em folha (14,1\% da produção mundial), atrás apenas da China. O Rio Grande do Sul é responsável por cerca de $50 \%$ da produção nacional. A cultura é típica de pequenas propriedades e a maior parte da produção localiza-se no entorno de indústrias de transformação e beneficiamento ${ }^{58}$. Esta característica econômica pode ter definido intrincadas relações entre produção agrícola e industrial, cultura e ações políticas (lobbies de produtores agrícolas e da indústria do tabaco) que podem ter influenciado o consumo mais elevado de tabaco pela população daquele Estado.

\section{Tabagismo passivo e câncer}

As suspeitas dos efeitos do tabagismo passivo sobre a saúde humana tiveram as primeiras citações em 1930, tendo-se firmado nos dias atuais pelas evidências verificadas e revisadas nas monografias da $\operatorname{IARC}^{40}$. Na urina de fumantes passivos foram encontradas concentrações variáveis de mutagênicos, derivados do benzopireno, nitrosaminas e outros componentes cancerígenos presentes na corrente secundária, e consequentemente submetendo esses indivíduos ao risco de contraírem câncer de pulmão ${ }^{59}$.

O Instituto de Pesquisa do Centro Nacional do Câncer do Japão realizou o primeiro grande estudo epidemiológico prospectivo que mostrou evidências conclusivas do dano causado pelo tabagismo passivo. Por 14 anos foram seguidas 95.540 mulheres com 40 anos ou mais de idade, não fumantes, casadas com maridos tabagistas ou não. A mortalidade por câncer de pulmão nas esposas de maridos consumidores de 20 cigarros/dia foi de 15,5 por $100 \mathrm{mil} \mathrm{mu-}$ lheres, quase o dobro da apurada naquelas cujos maridos não fumavam, ou seja, 8,7 por 100 mil mulheres ${ }^{60}$. Posteriormente, o autor verificou que os coeficientes de mortalidade por câncer de pulmão nas mulheres da coorte apresentavam gradiente positivo com o número de cigarros fumados por dia pelo cônjuge ${ }^{61}$.

Desde então, os resultados dos estudos japoneses têm sido confirmados por outros estudos internacionais. Por exemplo, pesquisa em cinco áreas metropolitanas nos 
Estados Unidos e 11 áreas em sete países da Europa Ocidental identificou claro efeito dose-resposta da exposição ao tabagismo ambiental tendo como referência o cônjuge fumante, exposição nos locais de trabalho e locais públicos com o desenvolvimento de câncer de pulmão em não fumantes ${ }^{62}$.

Além do câncer de pulmão, foi detectada a associação do tabagismo passivo, considerando-se longos períodos de exposição no domicílio e/ou nos ambientes de trabalho, com tumores de faringe e laringe entre indivíduos não fumantes ${ }^{63}$, embora tais evidências tenham sido consideradas apenas como limitadas na última revisão da IARC ${ }^{3}$.

Correa et al ${ }^{64}$ verificaram maior incidência de câncer de pulmão em indivíduos que foram expostos ao tabaco ambiental na infância devido ao tabagismo materno. No Reino Unido, entre crianças convivendo com pais fumantes foi detectado risco alto, mas sem significância estatística, de hepatoblastoma ${ }^{65}$. Também foi registrado um aumento de leucemias entre crianças com pais fumantes, especialmente leucemia linfocítica aguda ${ }^{66}$. Na Monografia 100 da IARC estas evidências foram assumidas como limitadas ${ }^{3}$.

Considerando-se que a corrente secundária contém a maioria dos constituintes da corrente primária, é provável que o tabagismo passivo possa estar associado a outros tipos de tumores. Novas pesquisas são necessárias para comprovação.

\section{Considerações finais}

Os inquéritos populacionais indicam avanços na política de controle do uso do tabaco no Brasil:

- a prevalência em todas as faixas etárias da população apresenta tendência decrescente no tempo;

- desde 2003 há acentuada queda do tabagismo nas faixas etárias mais jovens da população e entre as mulheres; e

- o consumo de cigarros per capita foi reduzido.
A mortalidade por todos os tipos de câncer no país tanto entre os homens como entre as mulheres está estabilizada ${ }^{67}$. Considerando-se que o tabaco vincula-se causalmente a vinte diferentes tipos de câncer, infere-se que o cenário de declínio da prevalência do tabagismo induzirá reduções na incidência e na mortalidade por câncer na população brasileira no horizonte das próximas décadas.

Do ponto de vista da pesquisa, estudos epidemiológicos devem ser conduzidos para definir a influência do tabagismo sobre o risco de ocorrência de neoplasias com evidências ainda limitadas de associação com o tabagismo, a exemplo do câncer de mama e da leucemia linfocítica aguda.

Do ponto de vista da saúde pública, apesar do cenário otimista observado em relação à prevalência, não deve ser esquecido que os 25 milhões de fumantes no país formam um contingente de proporção gigantesca e que funcionará como uma força para a manutenção da incidência de câncer relacionado ao tabaco no futuro. Desde sua implantação, em 1989, as ações do Programa Nacional de Controle do Tabagismo $(\mathrm{PNCT})^{10}$, coordenadas pelo INCA, órgão do Ministério da Saúde, em parceria com secretarias municipais e estatuais de saúde, bem como setores da sociedade civil organizada, parecem ter tido papel relevante na redução da prevalência de fumantes. Estas ações devem ser mantidas e aprofundadas.

Há ainda desafios a serem enfrentados. A epidemia tabágica no país apresenta distribuição desigual entre as classes sociais, atingindo de forma mais intensa as populações de mais baixa renda e com menor tempo de educação formal, portanto, mais permeáveis às mensagens da indústria do tabaco sem crivo crítico.

A prevalência do tabagismo entre adolescentes, apesar de decrescente no tempo, é sempre motivo de preocupação, pois formam a parcela da população mais vulnerável. A iniciação precoce ao uso de tabaco torna difícil sua interrupção na vida adulta. Neste sentido, as informações geradas pelo Vigescola ${ }^{20}$ exigem atenção 
das autoridades sanitárias. Entre os adolescentes de 12 e 16 anos entrevistados, cerca de $30 \%$ dos adolescentes referiram ter pelo menos um dos pais fumante. Por outro lado, $10 \%$ reportaram terem recebido ofertas de cigarros a título de propaganda por agentes das empresas produtoras de tabaco, e outros $5 \%$ informaram possuírem objetos pessoais com logomarcas de cigarros. É imperativo buscar alternativas para coibir tais práticas.
A constatação da presença de nicotina ambiental em hospitais e escolas do país é um exemplo da dimensão da tarefa a ser empreendida para tornar os ambientes livres da fumaça do tabaco. Esses são locais que devem servir de guia de conduta para a sociedade em relação ao tabaco, cujos princípios possam estender-se aos ambientes privados, onde é grave a situação da exposição involuntária à fumaça do tabaco.

\section{Referências}

1. WHO. WHO Report on the Global Tobacco Epidemic, 2009. Disponível em http://www.who.int/tobacco/ mpower/en/index.html [Acessado em 09 de dezembro de 2009].

2. Mackay J, Eriksen M. The tobacco atlas. Geneva: World Health Organization; 2002.

3. Secretan B, Straif K, Baan R, Grosse Y, El Ghissassi F, Bouvard V, et al. A review of human carcinogens - Part E: tobacco, areca nut, alcohol, coal smoke, and salted fish. Lancet Oncol 2009; 10: 1033-4.

4. Jha P, Chaloupka FJ. Curbing the epidemic: governments and the economics of tobacco control. Washington: World Bank; 1999.

5. IBGE. Instituto Brasileiro de Geografia e Estatística. Pesquisa Nacional por Amostra de Domicílios (PNAD). Tabagismo, 2008. Disponível em http://www.ibge.gov.br/ home/estatistica/ populacao/trabalhoerendimento/ pnad2008/suplementos/tabagismo/pnad-tabagismo. pdf [Acessado em 09 de dezembro de 2009].

6. Brasil. Ministério da Saúde. Instituto Nacional de Alimentação e Nutrição. Pesquisa nacional sobre saúde e nutrição. Brasília: INAN; 1990.

7. INCA. Instituto Nacional do Câncer. Secretaria de Vigilância em Saúde, Ministério da Saúde. Inquérito domiciliar sobre comportamentos de risco e morbidade referida de doenças e agravos não transmissíveis. Brasil, 15 capitais e Distrito Federal, 2002-2003. Rio de Janeiro: INCA; 2004.

8. Rego RA, Berardo FAN, Rodrigues SS, Oliveira ZM, Oliveira MB, Vasconcellos C, et al. Fatores de risco para doenças crônicas não-transmissíveis: inquérito domiciliar no Município de São Paulo, SP (Brasil). Metodologia e resultados preliminares. Rev Saúde Pública 1990; 24: 277-85.

9. Marcopito LF, Rodrigues SS, Pacheco MA. Prevalência de alguns fatores de risco para doenças crônicas na cidade de São Paulo. Rev Saúde Pública 2005; 39: 738-45.
10. Cavalcante T. Experiencia brasileña con políticas de control del tabaquismo. Salud Pub México 2004; 46: 54958.

11. Monteiro CA, Cavalcante TM, Moura EC, Claro RM, Szwarcwald CL. Population-based evidence of a strong decline in the prevalence of smokers in Brazil (19892003). Bull World Health Org 2007; 85: 527-34.

12. Weiss W. Cigarette smoking and lung cancer trends. A light at the end of the tunnel? Chest 1997; 111: 1414-6.

13. Everett SA, Warren CW, Sharp D, Kann L, Husten CG, Crossett LS. Initiation of cigarette smoking and subsequent smoking behavior among U.S. high school students. Prev Med 1999; 29: 327-33.

14. Burt RD, Dinh KT, Peterson Jr. AV, Sarason IG. Predicting adolescent smoking: a prospective study of personality variables. Prev Med 2000; 30: 134-45.

15. Simantov E, Schoen C, Klein JD. Health compromising behaviours: why do adolescents smoke or drink? Identifying underlying risk and protective factors. Arch Pediatr Adolesc Med 2000; 154: 1025-33.

16. Carvajal S, Wiatrek DE, Evans RI, Knee CR, Nash SG. Psychosocial determinants on the onset of escalation of smoking: cross sectional and prospective findings in multiethinic middle school samples. J Adolesc Health 2000; 27: 255-65.

17. Ariza C, Nebof M. Factors associated with smoking progression among Spanish adolescents. Health Educ Res 2002; 17: 750-60.

18. Malcon MC, Menezes AMB, Chatkin M. Prevalência e fatores de risco para tabagismo em adolescentes. Rev Saúde Pública 2003; 37: 1-7.

19. Guedes DP, Guedes JERP, Barbosa DS, Oliveira JA. Uso de tabaco e perfil lipídico-lipoprotéico plasmático em adolescentes. Rev Assoc Med Bras 2007; 53: 59-63. 
20. INCA. Instituto Nacional do Câncer. Secretaria de Vigilância em Saúde, Ministério da Saúde. Vigescola: vigilância do tabagismo em escolares. Dados e fatos de 17 cidades brasileiras. Disponível em http://www.inca. gov.br/vigescola/docs/vigescola/docs/vigescolafim1/ pdf. [Acessado em 09 de dezembro de 2009].

21. Whitlock G, MacMahon S, Vander Hoorn S, Davis P, Jackson R, Norton R. Socioeconomic distribution of smoking in a population of 10,529 New Zealanders. $N Z$ Med J 1997; 110: 327-30.

22. Soteriades ES, Difranza JR. Parent's socioeconomic status, adolescents' disposable income, and adolescents' smoking status in Massachusetts. Am J Public Health 2003; 93: 1155-60.

23. Federico B, Kunst AE, Vannoni F, Damiani G, Costa G. Trends in educational inequalities in smoking in northern, mid and southern Italy, 1980-2000. Prev Med 2004; 39: 919-26.

24. Villalbi JR, Tomas Z, Lopez MJ, Rodriguez M, Nebot M. Changing epidemiology of smoking: Barcelona 20002001. Rev Clin Esp 2004; 204: 312-6.

25. Fukuda Y, Nakamura K, Takano T. Socioeconomic pattern of smoking in Japan: income inequality and gender and age differences. Ann Epidemiol 2005; 15: 365-72.

26. Wen CP, Levy DT, Cheng TY, Hsu CC, Tsai SP. Smoking behaviour in Taiwan, 2001. Tob Control 2005; 14: 151-5.

27. Harman J, Graham H, Francis B, Inskip HM. Socioeconomic gradients in smoking among young women: A British survey. Soc Sci Med 2006; 63: 2791-800.

28. Khang YH, Cho HJ. Socioeconomic inequality in cigarette smoking: trends by gender, age, and socioeconomic position in South Korea, 1989-2003. Prev Med 2006; 42: 415-22.

29. Lolio CA, Pereira JC, Lotufo PA, De Souza JM. Hipertensão arterial e possíveis fatores de risco. Rev Saúde Pública 1993; 27: 357-62.

30. Moreira LB, Fuchs FD, Moraes RS, Bredemeir M, Cardozo S. Prevalência de tabagismo e fatores associados em área metropolitana da região sul do Brasil. Rev Saúde Pública 1995; 29: 46-51.

31. Lima-Costa MF. A escolaridade afeta igualmente comportamentos prejudiciais à saúde de idosos e adultos mais jovens? Inquérito de saúde da Região Metropolitana de Belo Horizonte, Minas Gerais, Brasil. Epidemiol Serv Saúde 2004; 13: 201-8.

32. César CLG, Carandina L, Alves MCP, Barros MBA, Goldbaum M. Saúde e condição de vida em São Paulo. Inquérito multicêntrico de saúde no Estado de São Paulo - ISA/SP. São Paulo: Universidade de São Paulo, Faculdade de Saúde Pública; 2005.

33. Szwarcwald CI, Viacava F, Vasconcellos MTL, Leal MC, Azevedo LO, Queiroz RSB, et al. O Brasil em números. RADIS 2004; 23: 14-33.
34. Azevedo e Silva G, Valente JV, Almeida LM, Moura EC, Malta DC. Tobacco smoking and level of education in Brazil, 2006. Rev Saúde Pública 2009; 43(S2): 48-56.

35. Caballero-Hidalgo A, González B, Pinilla J, Barber P. Factores predictores del inicio y consolidación del consumo de tabaco en adolescentes. Gaz Sanit 2005; 19: 440-7.

36. Hackshaw A, Law M, Wald NJ. The accumulated evidence on lung cancer and environmental tobacco smoke. BMJ 1997; 315: 980-8.

37. Mirra AP. Câncer e Tabagismo. In: Viegas C. A. Tabagismo: do diagnóstico à saúde pública. São Paulo: Atheneu; 2007, p. 107-16.

38. Araújo AJ. Tabagismo passivo. In: Viegas, C.A. Tabagismo: do diagnóstico à saúde pública. São Paulo: Atheneu; 2007, p. 37-75.

39. Barton HA, Cogliano J, Flowers L, Valcovic L, Woodrow S, Woodruff TJ. Assessing susceptibility from early-life exposure to carcinogens. Environ Health Perspect 2005; 113: 1125-33.

40. IARC. Tobacco smoke and involuntary smoking. IARC Monogr Eval Carcinog Risk Hum 2004; 83: 1-1438.

41. Mannino DM, Siegel M, Husten C, Rose D, Etzel R. Environmental tobacco smoke exposure and health effects in children: results from the 1991 National Health Interview Survey. Tob Control 1996; 5: 13-8.

42. Gerlach KK, Shopland DR, Hartman AM, Gibson JT, Pechacec TF. Workplace smoking policies in the United States: results from a national survey of more than 100.000 workers. Tob Control 1997; 6: 199-206.

43. Curtin F, Morabia A, Bernstein M. Lifetime exposure to environmental tobacco smoke among urban women: differences by socioeconomic class. Am J Epidemiol 1998; 148: 1040-7.

44. Whitlock G, MacMahon S, Vander Hoorn S, Davis P, Jackson R, Norton R. Association of environmental tobacco smoke exposure with socioeconomic status in a population of 7,725 New Zealanders. Tob Control 1998; 7: $276-80$.

45. Parsons WD, Carmella SG, Akerkar S, Bonilha LE, Hich SS. A metabolite of the tobacco-specific lung carcinogen 4-(methylnitrosamino)-1-(3-pyridyl)-1-butanone in the urine of hospital workers exposed to environmental tobacco smoke. Cancer Epidemiol Biomarkers Prev 1998; 7: $257-60$

46. Navas-Acien A, Peruga A, Breysse P, Zavaleta A, BlancoMarquizo A, Pitarque R, et al. Secondhand tobacco smoke in public places in Latin America, 2002-2003. JAMA 2004; 291: 2741-5

47. Lambert WE, Samet JM, Spengler JD. Environmental tobacco smoke concentrations in no-smoking and smoking sections of restaurants. Am J Public Health 1993; 83: 1339-41. 
48. Akbar-Khanzadeh F. Exposure to environmental tobacco in restaurants without separate ventilation systems for smoking and nonsmoking dining areas. Arch Environ Health 2003; 58: 97-102.

49. Doll R, Hill AB. Smoking and carcinoma of the lung: preliminary report. Br Med J 1950; 2: 739-48.

50. Doll R. Smoking and lung cancer. Proc R Soc Med 1957; 50: 503-4.

51. Doll R, Peto R. The causes of cancer: quantitative estimates of avoidable risks of cancer in the United States today. New York: Oxford University Press; 1981.

52. IARC. Tobacco smoking. IARC Monogr Eval Carcinog Risk Hum 1986; 38: 1-421.

53. Kurian AW, Balise RR, McGuire V, Whittemore AS. Histologic types of epithelial ovarian cancer: have they different risk factors? Gynecol Oncol 2005; 96: 520-30.

54. Liang PS, Chen TY, Giovannucci E. Cigarette smoking and colorectal cancer incidence and mortality: systematic review and meta-analysis. Int J Cancer 2009; 124: 2406-15.

55. Malta DC, Moura LM, Souza MFM, Curado MP, Alencar AP, Alencar GP. Tendência da mortalidade do câncer de pulmão, traquéia e brônquios no Brasil, 1980-2003. J Bras Pneumol 2007; 33: 536-43.

56. Curado MP, Edwards B, Shin HR, Storm H, Ferlay J, Heanue M, Boyle P. (Eds.). Cancer Incidence in Five Continents, Vol IX, IARC Sci Publ vol. 160. Lyon: IARC Press; 2007. Disponível em http://www.iarc.fr/en/ publications/pdfs-online/epi/sp160/index.php [Acessado em 09 de dezembro de 2009].

57. Brasil. Ministério da Saúde. Secretaria de Atenção à Saúde. Instituto Nacional do Câncer. Coordenação de Prevenção e Vigilância de Câncer. Estimativas 2008: Incidência de câncer no Brasil. Rio de Janeiro: INCA; 2007.
58. SEPLAG. Secretaria do Planejamento e Gestão. Departamento de Planejamento Governamental. Atlas Socioeconômico do Rio Grande do Sul. Disponível em http://www.rs.gov.br/atlas/ com base na edição impressa em 2002. [Acessado em 09 de dezembro de 2009].

59. Bos RP, Theuws JL, Henderson PT. Excretion of mutagens in human urine after passive smoking. Cancer Lett 1983; 19: 85-90.

60. Hirayama T. Non-smoking wives of heavy smokers have a higher risk of lung cancer: a study from Japan. $\mathrm{Br} \mathrm{Med} J$ 1981; 282: 183-5.

61. Hirayama T. Cancer mortality in non-smoking women with smoking husband based on a large-scale cohort study in Japan. Prev Med 1984; 13: 680-90.

62. Brennan P, Buffler PA, Reynolds P, Wu AH, Wichmann HE, Agudo A, et al. Secondhand smoke exposure in adulthood and risk of lung cancer among never smokers: a pooled analysis of two large studies. Int Cancer 2004; 109: 125-31.

63. Lee Y-C, Boffetta P, Sturgis EM, Wei Q, Zhang Z-F, Muscat J, et al. Involuntary smoking and the risk of head and neck cancer: pooled analysis of the INHANCE Consortium. Cancer Epidemiol Biomarkers Prev 2008; 17: 1974-81.

64. Correa P, Pickle LW, Fontham E, Haenszel W. Passive smoking and lung cancer. Lancet 1983; 2: 595-7.

65. Pang D, McNally R, Birch JM. Parental smoking and childhood cancer: results from the United Kingdom Childhood Cancer Study. Br J Cancer 2003; 88: 373-81.

66. Lee KM, Ward MH, Han S, Ahn HS, Kang HJ, Choi HS, et al. Paternal smoking, genetic polymorphisms in CYP1A1 and childhood leukemia risk. Leuk Res 2009; 33: 250-8.

67. Wünsch Filho V, Moncau JE. Mortalidade por câncer no Brasil 1980-1995: padrões regionais e tendências temporais. Rev Assoc Med Bras 2002; 48: 250-7.

Recebido em: 23/02/10 Aprovado em: 05/04/10 